REPRESENTATION THEORY

An Electronic Journal of the American Mathematical Society

Volume 11, Pages 1-15 (January 5, 2007)

S $1088-4165(07) 00298-1$

\title{
ON THE UNIQUENESS OF FOURIER JACOBI MODELS FOR REPRESENTATIONS OF $U(n, 1)$
}

\author{
EHUD MOSHE BARUCH AND STEPHEN RALLIS
}

\begin{abstract}
We show that every irreducible unitary representation of $U(n, 1)$, has at most one Fourier Jacobi model.
\end{abstract}

\section{INTRODUCTION}

Fourier Jacobi coefficients and Fourier Jacobi models arise in the expansion of automorphic forms on reductive groups $G$ with a Heisenberg parabolic. A Heisenberg parabolic is a parabolic subgroup whose unipotent radical is a Heisenberg group. The expansion is in terms of Jacobi forms which are certain automorphic forms on this parabolic subgroup. The coefficients of these Jacobi forms are called Fourier Jacobi coefficients. (For the classical setting of Siegel modular forms expanded using Jacobi forms see [3].) When this is done in an adelic setting ([5], 6], [13]), the expansion leads to the Fourier Jacobi models which are certain induced spaces on which the group $G$ acts. A central ingredient in this approach is the conjectural multiplicity free property of this induced space. This is equivalent to a unique embedding of certain irreducible unitary representations into this space. Such an embedding is called a Fourier Jacobi model for the given irreducible unitary representation. In this paper we consider the case where $G=U(n, 1)=U(n, 1)(\mathbb{R})$, a real reductive group of rank one. The Heisenberg parabolic is the minimal (and only) parabolic of $G$ and we prove this uniqueness results for general Fourier Jacobi models. Such results were obtained for certain classes of representations in ([12, [10, [9], 8], 7]). Our method of proof, using invariant distributions as in the Whittaker case [15], generalizes the result of [1] for the group $U(2,1)$. (A similar $p$-adic result for $S p(4)$ was obtained in [2]). Many of the ideas and techniques are the same as in 11. The main difference is that in general, the Levi subgroup of our parabolic is nonabelian and is isomorphic to the compact group $U(n-1) \times U(1)$. Hence we need to apply an induction process on centralizers of semisimple elements in $U(n-1)$ that did not appear in [1. In particular, we prove a new result on invariant distributions on $U(n) \times \mathbb{C}^{n}$ which we think is interesting by itself. This result is an analog of results of the second author in the $p$-adic case.

Correction of Error: In 2] and [1] the uniqueness property is stated for irreducible admissible representations. The proof, however, holds only for irreducible

Received by the editors October 28, 2005, and in revised form, September 18, 2006.

2000 Mathematics Subject Classification. Primary 22E50; Secondary 11F70.

Key words and phrases. Fourier Jacobi, invariant distributions.

Research of the second author was partially supported by the NSF.

(C)2007 American Mathematical Society 
admissible unitary representations and should have been stated for these representations.

\section{The MAIN RESUlT}

Let $I_{k}$ be the identity matrix of order $k \times k$. Let

$$
U(n)=\left\{A \in G L_{n}(\mathbb{C}): \bar{A}^{t} A=I_{n}\right\} \text { and } w=\left(\begin{array}{ccc}
0 & 0 & 1 \\
0 & I_{n-1} & 0 \\
1 & 0 & 0
\end{array}\right) .
$$

Let $G=U(n, 1)$ be defined by $G=\left\{A \in G L_{n+1}(\mathbb{C}): \bar{A}^{t} w A=w\right\}$. Let $N$ be a Heisenberg in $G$ defined by

$$
N=\left\{\left(\begin{array}{ccc}
1 & u & z \\
0 & 1 & -\bar{u}^{t} \\
0 & 0 & 1
\end{array}\right): u \in \mathbb{C}^{n-1}, z \in \mathbb{C}, z+\bar{z}=-u \bar{u}^{t}\right\} .
$$

The center of $N$ is

$$
Z=\left\{\left(\begin{array}{lll}
1 & 0 & z \\
0 & 1 & 0 \\
0 & 0 & 1
\end{array}\right): z \in \mathbb{C}, z+\bar{z}=0\right\}
$$

Let

$$
M=\left\{d(a, X)=\left(\begin{array}{ccc}
a & 0 & 0 \\
0 & X & 0 \\
0 & 0 & \bar{a}^{-1}
\end{array}\right): a \in \mathbb{C}^{*}, X \in U(n-1)\right\}
$$

and

$$
S=\left\{d(1, X)=\left(\begin{array}{ccc}
1 & 0 & 0 \\
0 & X & 0 \\
0 & 0 & 1
\end{array}\right): X \in U(n-1)\right\}
$$

Let $P=M N$ be a minimal parabolic of $G$ and let $J=S N$ be a Fourier Jacobi subgroup of $G$. We have that $G=P \bigcup P w P$.

Let $\psi$ be a nontrivial character of $Z$ and let $\theta_{\psi}$ be the oscillator representation of $N$ with central character $\psi$. We shall use the Schrödinger model (see $([11,3.1)$ or [4]) for $\theta_{\psi}$. The smooth part of $\theta_{\psi}$ can be identified with $S\left(\mathbb{R}^{n-1}\right)$ which is the space of Schwartz functions on $\mathbb{R}^{n-1}$. We put on $S\left(\mathbb{R}^{n-1}\right)$ the usual Frechét topology.

The representation which is contragredient to the oscillator representation with central character $\psi$ can be identified with $\theta_{\psi^{-1}}$. It is well known that $\theta_{\psi}$ can be extended to an irreducible unitary representation of $J$. Let $\sigma$ be an irreducible unitary representation of $U(n-1)$ on a finite dimensional vector space $V_{\sigma}$ which we view as a representation of $S$. We extend $\sigma$ to $J$ by letting $N$ act trivially. Then $\sigma \otimes \theta_{\psi}$ is an irreducible unitary representation of $J$.

Let $(\pi, H)$ be an irreducible unitary representation of $G$ on a Hilbert space $H$. Let $H_{\infty}$ be the smooth part of $H$. Our main result in this paper is the following.

Theorem 2.1.

$$
\operatorname{dim}\left(\operatorname{Hom}_{J}\left(\pi, \sigma \otimes \theta_{\psi}\right)\right) \leq 1
$$

Remark 2.2. Here $\operatorname{Hom}_{J}$ denotes the space of continuous linear $J$ invariant maps between the Frechét spaces $H_{\infty}$ and $V_{\sigma} \otimes S\left(\mathbb{R}^{n-1}\right)$. 
If the dimension of the above Hom space is one, then $\pi$ can be embedded in the space

$$
\operatorname{Ind}_{J}^{G}\left(\sigma \otimes \theta_{\psi}\right) .
$$

We call this unique embedding, a Fourier Jacobi model for $\pi$ corresponding to the Fourier Jacobi data $(\sigma, \psi)$.

In order to prove Theorem 2.1 we notice that there is a natural injection from $\operatorname{Hom}_{J}\left(\pi, \sigma \otimes \theta_{\psi}\right)$ to $\operatorname{Hom}_{J} \Delta\left(\pi \otimes\left(\chi \otimes \theta_{\psi}\right)^{\vee}, 1\right)$ where $\pi \otimes\left(\sigma \otimes \theta_{\psi}\right)^{\vee}$ is a representation of $G \times J, \quad J^{\triangle}$ is the diagonal embedding of $J$ into $G \times J$ and $\left(\sigma \otimes \theta_{\psi}\right)^{\vee}$ is the representation which is $J$ contragredient to $\sigma \otimes \theta_{\psi}$. By the remarks above on the oscillator representation we have that

$$
\left(\sigma \otimes \theta_{\psi}\right)^{\vee}=\check{\sigma} \otimes \theta_{\psi^{-1}} .
$$

Hence

$$
\operatorname{dim}\left(\operatorname{Hom}_{J}\left(\pi, \sigma \otimes \theta_{\psi}\right)\right) \leq \operatorname{dim}\left(\operatorname{Hom}_{J} \Delta\left(\pi \otimes\left(\check{\sigma} \otimes \theta_{\psi^{-1}}\right), 1\right)\right) .
$$

Thus, Theorem 2.1 will follow from

Theorem 2.3.

$$
\operatorname{dim}\left(\operatorname{Hom}_{J} \triangle\left(\pi \otimes\left(\sigma \otimes \theta_{\psi}\right), 1\right)\right) \leq 1 .
$$

for every irreducible unitary representations $\pi$ of $G$ and $\sigma$ of $S$ and every nontrivial character $\psi$ of $Z$.

To prove Theorem 2.3 we will need the following: Let $Q=G \times J$ and let $\tau$ be an anti-involution on $Q$ defined by

$$
\tau(g, j)=\left(\bar{g}^{-1}, \bar{j}^{-1}\right) .
$$

For a function $f \in C_{c}^{\infty}(Q)$ we let $\left(\rho_{l}(q) f\right)(x)=f\left(q^{-1} x\right),\left(\rho_{r}(q)\right) f(x)=f(x q)$ and $f^{\tau}(x)=f(\tau(x))$. If $D$ is a distribution on $Q$, then we define $\left(\rho_{l}(q) D\right)(f)=$ $D\left(\rho_{l}\left(q^{-1}\right) f\right),\left(\rho_{r}(q) D\right)(f)=D\left(\rho_{r}(q) f\right)$ and $D^{\tau}(f)=D\left(f^{\tau}\right)$. Let $\square$ be the Casimir differential operator associated to $G$. Then $\square \otimes 1$ is a differential operator on $Q$ that acts on the $G$ variable in $Q$. The main result that we need in order to prove Theorem 2.3 is:

Theorem 2.4. Let $D$ be a distribution on $Q$. Assume that

(a) $\rho_{l}(j) D=D=\rho_{r}(j) D, \quad j \in J^{\triangle}$.

(b) $\rho_{r}(e, z) D=\psi(z) D, \quad z \in Z$.

(c) $(\square \otimes 1) D=\beta D$ for some scalar $\beta \in \mathbb{C}$.

Then $D^{\tau}=D$.

This theorem will imply Theorem 2.3 as in (15], pp. 183-185). For the sake of completeness we repeat the proof here. Our version of the proof is slightly different than in [15. We recommend that the reader skip the next section and return to it only if it is needed.

\section{Proof of Theorem 2.3 from Theorem 2.4}

The argument goes as follows: We denote by $\Pi=\Pi_{\pi, \sigma, \psi}=\pi \otimes\left(\sigma \otimes \theta_{\psi}\right)$ the irreducible unitary representation of $Q=G \times J$ on a Hilbert space $H_{\Pi}$ obtained as above. We let $H_{\Pi}^{\infty}=\left\{\Pi(f) v: f \in C_{c}^{\infty}(Q), v \in H_{\Pi}\right\}$ with the usual Frechét topology which is defined as follows. Let $\mathfrak{q}=\operatorname{Lie}(Q)$. Let $U(\mathfrak{q})$ be the universal enveloping algebra of $\mathfrak{q}$. For every $Y \in U(\mathfrak{q})$ we define a seminorm $\alpha_{Y}$ on $H_{\Pi}^{\infty}$ by $\alpha_{Y}(v)=\|Y(v)\|, v \in H_{\Pi}^{\infty}$. Then the topology is given by this set of seminorms. 
The contragredient representation $\check{\Pi}=\Pi_{\check{\pi}, \check{\sigma}, \psi^{-1}}$ is defined on $H_{\Pi}^{*}$. That is, if $L$ is a continuous functional on $H_{\Pi}$, then we set

$$
(\check{\Pi}(q) L)(v)=L\left(\Pi\left(q^{-1}\right) v\right), \quad v \in H_{\Pi} .
$$

Since $\Pi$ is unitary we can identify the representation $\check{\Pi}$ with the representation $\bar{\Pi}$ which is defined on $\bar{H}_{\Pi}$. Here $\bar{H}_{\Pi}$ is a vector space which is identified as an additive group with $H_{\Pi}$. Scalar multiplication is defined by $\lambda(v)=\bar{\lambda} v$ where $v \in H, \lambda \in \mathbb{C}$. The action of $Q$ on $\bar{H}_{\Pi}$ is defined by $\bar{\Pi}(q) v=\Pi(q) v$. If $\langle u, v\rangle$ is a $Q$ invariant inner product on $H_{\Pi}$, then $\overline{\langle u, v\rangle}=\langle v, u\rangle$ is a $Q$ invariant inner product on $\bar{H}_{\Pi}$.

Let $L$ be a continuous functional on $H_{\Pi}^{\infty}$. For $f \in C_{c}^{\infty}(Q)$ we let $\check{\Pi}(f) L$ be a functional on $H_{\Pi}$. That is, let $\check{f}(q)=f\left(q^{-1}\right)$. Then

$$
(\check{\Pi}(f) L)(u)=L(\Pi(\check{f}) u), \quad u \in H_{\Pi} .
$$

Lemma 3.1 ([15], Proposition 3.2). $\check{\Pi}(f) L$ is continuous on $H_{\Pi}$.

Proof. Let $v_{n} \in H_{\Pi}$ and assume $\left\|v_{n}\right\| \rightarrow 0$. Then $\alpha\left(\Pi(\check{f}) v_{n}\right) \rightarrow 0$ for every seminorm $\alpha$ that defines the topology on $H_{\Pi}^{\infty}$, hence $\Pi(\check{f}) v_{n} \rightarrow 0$ in $H_{\Pi}^{\infty}$. Since $L$ is continuous on $H_{\Pi}^{\infty}$ it follows that $L\left(\Pi(\check{f}) v_{n}\right) \rightarrow 0$ which means that $(\check{\Pi}(f) L)\left(v_{n}\right) \rightarrow$ 0 and $\check{\Pi}(f) L$ is bounded on $H_{\pi}$.

Let $\langle$,$\rangle be a fixed Q$ invariant inner product on $H_{\Pi}$. Then by the above lemma there exists a unique $v=v_{\check{\Pi}(f) L} \in H_{\Pi}$ such that

$$
(\check{\Pi}(f) L)(u)=\left\langle u, v_{\check{\Pi}(f) L}\right\rangle \text { for all } u \in H_{\Pi} .
$$

Let $\bar{f}(g)=\overline{f(g)}$. If $v \in H_{\Pi}=\bar{H}_{\Pi}$, then $\Pi(\bar{f})(v)=\bar{\Pi}(f)(v)$. If $L$ is a continuous functional on $H_{\Pi}^{\infty}$, then $\bar{L}$ is a continuous functional on $\bar{H}_{\Pi}^{\infty}$. It is easy to see that

$$
v_{\check{\Pi}(f) L}=v_{\check{\Pi}(f) \bar{L}} .
$$

Lemma 3.2. Let $f_{1}, f_{2} \in C_{c}^{\infty}(Q), L$ a continuous functional on $H_{\Pi}^{\infty}$, and $\alpha$ a continuous functional on $\bar{H}_{\Pi}^{\infty}$. Then

(a) $v_{\check{\Pi}\left(f_{1} * f_{2}\right) L}=\Pi\left(\bar{f}_{1}\right) v_{\check{\Pi}\left(f_{2}\right) L}$.

(b) $\alpha\left(v_{\check{\Pi}\left(f_{1} * f_{2}\right) L}\right)=\left\langle v_{\check{\Pi}\left(\check{f}_{1}\right) \alpha}, v_{\check{\Pi}\left(f_{2}\right) L}\right\rangle$.

Proof. (a) Let $u \in H_{\Pi}$. Then

$$
\begin{aligned}
\left\langle u, v_{\check{\Pi}\left(f_{1} * f_{2}\right) L}\right\rangle & =\int_{Q} f_{1} * f_{2}(q) L\left(\Pi\left(q^{-1}\right) u\right) d q \\
& =\int_{Q} \int_{Q} f_{1}\left(q x^{-1}\right) f_{2}(x) L\left(\Pi\left(q^{-1}\right) u\right) d x d q \\
& =\int_{Q} f_{2}(x) \int_{Q} L\left(f_{1}\left(q x^{-1}\right) \Pi\left(q^{-1}\right) u\right) d q d x \\
& =\int_{Q} f_{2}(x) L\left(\int_{Q} f_{1}(q) \Pi\left(x^{-1} q^{-1}\right) u d q\right) d x \\
& =\int_{Q} f_{2}(x) L\left(\Pi\left(x^{-1}\right) \int_{Q} f_{1}(q) \Pi\left(q^{-1}\right) u d q\right) d x \\
& =\left(\check{\Pi}\left(f_{2}\right) L\right)\left(\Pi\left(\check{f}_{1}\right) u\right) \\
& =\left\langle\Pi\left(\check{f}_{1}\right) u, v_{\check{\Pi}\left(f_{2}\right) L}\right\rangle \\
& =\left\langle u, \Pi\left(\bar{f}_{1}\right) v_{\check{\Pi}\left(f_{2}\right) L}\right.
\end{aligned}
$$


(b)

$$
\begin{aligned}
\alpha\left(v_{\check{\Pi}\left(f_{1} * f_{2}\right) L}\right) & =\alpha\left(\Pi\left(\bar{f}_{1}\right) v_{\check{\Pi}\left(f_{2}\right) L}\right) \\
& =\check{\Pi}\left(\check{f}_{1}\right) \alpha\left(v_{\check{\Pi}\left(f_{2}\right) L}\right) \\
& =\left\langle v_{\check{\Pi}\left(\check{f}_{1}\right) \alpha}, v_{\check{\Pi}\left(f_{2}\right) L}\right\rangle
\end{aligned}
$$

Let $L$ be a nonzero continuous $J^{\triangle}$ invariant functional on $H_{\Pi}^{\infty}$. Let $\alpha$ be a nonzero continuous $J^{\triangle}$ invariant functional on $\bar{H}_{\Pi}^{\infty}$. (For example $\alpha=\bar{L}$.) For $f \in C_{c}^{\infty}(Q)$ we set

$$
D_{\alpha, L}(f)=\alpha\left(v_{\check{\Pi}(f) L}\right) .
$$

Lemma 3.3. $D_{\alpha, L}$ is a distribution. It satisfies conditions (a), (b), (c) of Theorem 2.4. (Hence it is invariant under $\tau$ ).

Proof. We first prove that $D_{\alpha, L}$ is a distribution. To do that we will show that if $f_{n} \in C_{c}^{\infty}(Q)$ and $f_{n} \rightarrow 0$, then $D_{\alpha, L}\left(f_{n}\right) \rightarrow 0 . D_{\alpha, L}\left(f_{n}\right)=\alpha\left(v_{\check{\Pi}\left(f_{n}\right) L}\right)$. Since $\alpha$ is continuous it is enough to show that $v_{\check{\Pi}\left(f_{n}\right) L} \rightarrow 0$ in $H_{\pi}^{\infty}$. To show that we will show that $\alpha_{Y}\left(v_{\breve{\Pi}\left(f_{n}\right) L}\right) \rightarrow 0$ for every $Y \in U(\mathfrak{q}) . \quad \alpha_{Y}\left(v_{\check{\Pi}\left(f_{n}\right) L}\right)=|| Y v_{\check{\Pi}\left(f_{n}\right) L} \mid=$ $\left\|v_{\check{\Pi}\left(Y f_{n}\right) L}\right\|$ Since $f_{n} \rightarrow 0$, we have that $Y f_{n} \rightarrow 0$ hence $\check{\Pi}\left(Y f_{n}\right) L \rightarrow 0$ and $v_{\check{\Pi}\left(f_{n}\right) L} \rightarrow 0$.

If $j \in J^{\triangle}$, then $v_{\check{\Pi}\left(\rho_{l}(j)(f)\right) L}=\Pi(j) v_{\check{\Pi}(f) L}$ and $v_{\check{\Pi}\left(\rho_{r}(j)(f)\right) L}=v_{\check{\Pi}(f) L}$ where $\rho_{l}, \rho_{r}$ are left and right translations respectively. It follows that $D_{\alpha, L}$ is invariant on the left and right by $J^{\triangle}$. Also if $z \in Z$, then $v_{\check{\Pi}\left(\rho_{r}((1, z))(f)\right) L}=\psi^{-1}(z) v_{\check{\Pi}(f) L}$ hence $D_{\alpha, L}$ is $((1, Z), \psi)$ equivariant. The action of the Casimir on the left variable is also clear.

Define another representation $\Pi^{*}$ of $Q$ on $H_{\Pi}$ by $\Pi^{*}(q)=\Pi(\bar{q}), q \in Q$. Here $\bar{q}$ is defined as follows: If $q=(g, j)$, then $\bar{q}=(\bar{g}, \bar{j})$. For $f \in C_{c}^{\infty}(Q)$ we define $f^{*}(q)=f(\bar{q})$. It is easy to see that $\Pi^{*}(f)=\Pi\left(f^{*}\right)$. We will show as in [15] that $\bar{\Pi}$ is equivalent to $\Pi^{*}$.

Lemma 3.4. Let $L$ and $\alpha$ be as in the theorem above. Let $f_{1}, f_{2} \in C_{c}^{\infty}(Q)$. Then

$$
\left\langle v_{\check{\Pi}\left(f_{1}^{*}\right) \alpha}, v_{\check{\Pi}\left(f_{2}\right) L}\right\rangle=\left\langle v_{\check{\Pi}\left(f_{2}^{*}\right) \alpha}, v_{\check{\Pi}\left(f_{1}\right) L}\right\rangle .
$$

Proof. By Lemma 3.3 and Theorem 2.3 we have that $D(f)=D\left(f^{\tau}\right)$. Applying this to $f=f_{1}^{\tau} * f_{2}$ we get that $D\left(f_{1}^{\tau} * f_{2}\right)=D\left(f_{2}^{\tau} * f_{1}\right)$. Since $\left(f^{\tau}\right)^{\tau}=f^{*}$ we can apply Lemma 3.2 (b) to get the result.

Remark 3.5. Let $K_{1}=U(n) \times U(1)$ be a maximal compact subgroup in $G$ and let $K_{2}=U(n-1)$ be a subgroup of $J$. Let $K=K_{1} \times K_{2}$ be a compact subgroup in $Q$. Let $\left(\Pi, H_{\Pi}\right)$ be a representation of $Q$ obtained as above. It is clear that the set of $K$ finite vectors in $H_{\Pi}$ is dense in $H_{\Pi}$. It follows that the set of vectors of the form $\Pi(f) v$, where $f \in C_{c}^{\infty}(Q)$ and $v \in H_{\Pi}$ is nonzero and dense in $H_{\Pi}$.

Theorem 3.6. Assume that $H_{\Pi}^{\infty}$ has a nonzero continuous $J^{\triangle}$ invariant functional L. Then $\bar{\Pi}$ is equivalent to $\Pi^{*}$.

Proof. Let $W=\left\{v_{\check{\Pi}(f) L}: f \in C_{c}^{\infty}(Q)\right\} . \quad W$ is nonzero otherwise $\check{\Pi}(f) L=0$ for every $f \in C_{c}^{\infty}(Q)$ hence $L(\Pi(\check{f}) v)=0$ for every $v \in H_{\Pi}$ and every $f \in$ $C_{c}^{\infty}(Q)$. Thus, it follows from the remark above that $L=0$, a contradiction. Since 
$v_{\check{\Pi}\left(\rho_{l}(q) f\right) L}=\bar{\Pi}(q) v_{\check{\Pi}(f) L}$, it follows that $W$ is a dense $\bar{\Pi}$ invariant subspace of $\bar{H}_{\Pi}$. We define a mapping $I: W \rightarrow H_{\Pi}$ by $I\left(v_{\check{\Pi}(f) L}\right)=v_{\check{\Pi}\left(f^{*}\right) \bar{L}}$. By choosing $\alpha=\bar{L}$, and $f_{1}^{*}=f_{2}$ in (3.2) we get that

$$
\left\|v_{\check{\Pi}\left(f_{2}\right) L}\right\|^{2}=\left\langle v_{\check{\Pi}\left(f_{2}\right) L}, v_{\check{\Pi}\left(f_{2}\right) L}\right\rangle=\left\langle v_{\check{\bar{\Pi}}\left(f_{2}^{*}\right) \bar{L}}, v_{\check{\Pi}\left(f_{2}^{*}\right) \bar{L}}\right\rangle=\|\left. v_{\check{\Pi}\left(f_{2}^{*}\right) \bar{L}}\right|^{2} .
$$

This implies that $I$ is well defined and that $I$ preserves norms. It is also easy to see that $I$ intertwines $\bar{\Pi}$ and $\Pi^{*}$, that is, $I(\bar{\Pi}(q) w)=\Pi^{*}(q)(I(w))$ for every $q \in Q, w \in W$. Hence $I$ extends to a unitary $G$ isomorphism between $H_{\bar{\Pi}}=\bar{H}_{\Pi}$ and $H_{\Pi^{*}}=H_{\Pi}$.

We will also need the following property of $I$ which follows from (3.2):

$$
\langle I(v), w\rangle=\langle v, I(w)\rangle
$$

for every $v, w \in H_{\bar{\Pi}}=H_{\Pi}$.

3.1. Proof of Theorem 2.3. Let $\left(\Pi, H_{\Pi}\right)$ be as above and assume that $H_{\Pi}^{\infty}$ has a nonzero continuous $J^{\triangle}$ invariant functional $L$. Let $\alpha$ be a nonzero continuous $J^{\triangle}$ invariant functional on $\bar{H}_{\Pi}^{\infty}$. We will prove that the vector $v_{\check{\Pi}(f) L}$ is proportional (with the same proportionality constant) to the vector $v_{\check{\Pi}\left(f^{*}\right) \alpha}$ for every $f \in C_{c}^{\infty}(Q)$. This means that $\alpha$ is determined by $L$ up to a constant, hence $\alpha=c \bar{L}$ for some constant $c$. If there was another linearly independent $J^{\triangle}$ invariant functional $L_{1}$ on $H_{\Pi}^{\infty}$, then we could take $\alpha=\overline{L_{1}}$ which is a contradiction to the uniqueness of $\alpha$.

Let $W^{\prime}=\left\{v_{\check{\Pi}\left(f^{*}\right) \alpha}: f \in C_{c}^{\infty}(Q)\right\}$. $W^{\prime}$ is $\Pi^{*}$ invariant and dense in $H_{\Pi}$. We define a map $R: W^{\prime} \rightarrow \bar{H}_{\Pi}$ by $R\left(v_{\check{\Pi}\left(f^{*}\right) \alpha}\right)=v_{\check{\Pi}(f) L}$. Using (3.2) it is easy to show that $R$ is well defined. It is easy to see that $R$ is an $Q$ invariant linear mapping between $\left(\Pi^{*}, W^{\prime}\right)$ and $\left(\bar{\Pi}, \bar{H}_{\Pi}\right)$. By (3.2) it satisfies

$$
\langle R u, v\rangle=\langle u, R v\rangle, \quad u, v \in W^{\prime} .
$$

We let $T=I \circ R$. Then $T$ is a $\Pi^{*}$ invariant linear map from $W^{\prime}$ to $H_{\Pi}$. We let $S=R \circ I$. Then $S$ is linear map from $I^{-1}\left(W^{\prime}\right)$ (which we think of as a subspace of $\left.H_{\Pi}\right)$ to $H_{\Pi}$. By (3.3) and (3.4) we have that

$$
\langle T u, v\rangle=\langle u, S v\rangle, \quad u \in W^{\prime}, v \in I^{-1}\left(W^{\prime}\right) .
$$

Hence, by [16], Proposition 1.2.2 applied with $D=W^{\prime}, D^{\prime}=I^{-1}\left(W^{\prime}\right)$ we have that $T$ is a multiple of the identity. It follows that $\alpha$ is determined by $L$ up to a scalar.

\section{Preliminaries}

4.1. Group actions. Let $X$ be a real analytic manifold. We denote by $C_{c}^{\infty}(X)$ the space of compactly supported and smooth functions on $X$. If a Lie group $G$ acts smoothly on $X$, then $G$ acts on $C_{c}^{\infty}(X)$ by

$$
g(\phi(x))=\phi\left(g^{-1} x\right), \quad g \in G, x \in X, \phi \in C_{c}^{\infty}(X) .
$$

In particular, if $X$ is a subset of $G$ and if $x \in X$ and $g \in G$, then we denote

$$
\begin{aligned}
& \rho_{l}(g)(x)=g x, \\
& \rho_{r}(g)(x)=x g^{-1}, \\
& g(x)=g x g^{-1} .
\end{aligned}
$$

$G$ acts on distributions by duality. 
We let $G=U(n, 1)$ and $\mathfrak{g}=\operatorname{Lie}(G)$ be the Lie Algebra of $G$ given by

$$
\mathfrak{g}=\left\{A \in M(n, \mathbf{C}): \bar{A}^{t} w+w A=0\right\} .
$$

$\mathfrak{g}$ acts on $C_{c}^{\infty}(G)$ by left invariant (resp. right invariant) differential operators as follows. Let $\phi \in C_{c}^{\infty}(G), x \in G, A \in \mathfrak{g}$. We denote

$$
\begin{aligned}
& \left(L_{A} \phi\right)(x)=\left.\frac{d}{d t} \phi\left(e^{t A} x\right)\right|_{t=0}, \\
& \left(R_{A} \phi\right)(x)=\left.\frac{d}{d t} \phi\left(x e^{t A}\right)\right|_{t=0} .
\end{aligned}
$$

These actions extend to the universal enveloping algebra of $G$. Let $\square$ be the Casimir element in the universal enveloping algebra. Then $L_{\square}$ is defined as above.

4.2. An equivalent statement of the main result. Our main theorem, Theorem 2.4 is about invariant distributions. The main tools for studying these invariant distributions are Harish-Chandra's submersive maps and Frobenius reciprocity. A rough and short statement of these principles together with references to the precise statements can be found in ([2, Lemma 2.3 and Lemma 2.2).

For $g \in G$ we let $\tau(g)=\bar{g}^{-1}$. Applying Frobenius reciprocity (see [2, Theorem 2.5 and Theorem 2.6 for a similar situation) to the space of invariant distributions satisfying the conditions of Theorem 2.4 we get that Theorem 2.4 is equivalent to

Theorem 4.1. Let $T$ be a distribution on $G$. Assume that

(a) $j(T)=T, j \in J$.

(b) $\rho_{l}(z) T=\psi(z) T, \quad z \in Z$.

(c) $L_{\square} T=\beta T$ for some scalar $\beta \in \mathbf{C}$.

Then $T^{\tau}=T$.

Notice that the action of $j$ on $T$ denoted by $j(T)$ above is the action induced by conjugation. To prove Theorem 4.1 we will assume that $T$ is a distribution on $G$ satisfying (a), (b), (c) above and that $T$ is skew invariant under $\tau$, that is, $T^{\tau}=-T$, and we will show that $T=0$.

\section{INVARIANT DISTRIBUTIONS ON $U(n) \times \mathbb{C}^{n}$}

Our strategy for the proof of Theorem 4.1 is to restrict our skew invariant distribution $T$ to the open cell of $G$ and to show that it vanishes there. This will lead us to invariant distributions on $U(n-1) \times \mathbb{C}^{n-1}$ which we now describe.

The group $U(n)$ acts on the space $\mathbb{C}^{n}$ via the standard representation. That is, if $A \in U(n)$ is a unitary matrix and $v \in \mathbb{C}^{n}$ is a column vector, then the action is matrix multiplication. $U(n)$ acts on $U(n) \times \mathbb{C}^{n}$ via the action

$$
g(A, v)=\left(g A g^{-1}, g v\right) .
$$

Let $Y$ be a smooth manifold. We extend this action to $U(n) \times \mathbb{C}^{n} \times Y$ by letting $U(n)$ act trivially on $Y$. That is,

$$
g(A, v, y)=\left(g A g^{-1}, g v, y\right), \quad g, A \in U(n), v \in \mathbb{C}^{n}, y \in Y .
$$

We define an involution $\tau$ on $U(n) \times \mathbb{C}^{n} \times Y$ by

$$
\tau(A, v, y)=\left(\bar{A}^{-1},-\bar{v}, y\right) .
$$

Our main theorem of this section is the following: 
Theorem 5.1. Let $Q$ be a distribution on $U(n) \times \mathbb{C}^{n} \times Y$ and assume that $Q$ is invariant under the action of $U(n)$. Then $Q^{\tau}=Q$.

We will prove this theorem by an induction process using centralizers of elements in $U(n)$ as in Harish-Chandra's regularity theorem. To do that we will need a more general statement. We let $n_{1}, n_{2}, \ldots, n_{k}$ be positive integers and let $H=$ $H\left(n_{1}, n_{2}, \ldots, n_{k}\right)=U\left(n_{1}\right) \times U\left(n_{2}\right) \times \ldots \times U\left(n_{k}\right)$. If $h \in H$, then the centralizer of $h, C(h)$ is of the form $H\left(r_{1}, \ldots, r_{l}\right)$ with the semisimple rank of $H\left(r_{1}, \ldots, r_{l}\right)$ less than or equal to the semisimple rank of $H=H\left(n_{1}, n_{2}, \ldots, n_{k}\right)$ and equality holds if and only if $h$ is a central element in $H$. (The semisimple rank of $H$ is $\left.n_{1}+\ldots+n_{k}-k\right)$. Let $V=\mathbb{C}^{n_{1}} \times \mathbb{C}^{n_{2}} \times \ldots \times \mathbb{C}^{n_{k}}$. Then $H$ acts naturally on $V$ extending the above action of $U(n)$ on $\mathbb{C}^{n}$. We extend (5.1) to an action of $H$ on $H \times V \times Y$. We also extend the involution $\tau$ to $H \times V \times Y$. We shall prove the following:

Theorem 5.2. Let $Q$ be a distribution on $H \times V \times Y$ and assume that $Q$ is invariant under the action of $H$. Then $Q^{\tau}=Q$.

We first consider the case where the semisimple rank of $H$ is zero, that is, $n_{1}=$ $n_{2}=\ldots=n_{k}=1$. In that case, the action of $H$ is trivial on $H$ and the involution is trivial on $H$, hence we can move $H$ into $Y$. Therefore, our theorem reads:

Theorem 5.3. Let $Y$ be a smooth manifold and let $Q$ be a distribution on $\mathbb{C}^{n} \times Y$. Let $H=(U(1))^{n}$ act on $\mathbb{C}^{n}$ and on $\mathbb{C}^{n} \times Y$ as above. Assume that $Q$ is invariant under this action. Then $Q$ is invariant under the involution $\tau$ where $\tau(v, y)=$ $(-\bar{v}, y), v \in \mathbb{C}^{n}, y \in Y$.

When $n=1$, that is, $Q$ is a distribution on $\mathbb{C} \times Y$, this theorem is proved in ([1], Lemma 4.2). The general case is similar. We prove here the case $n=2$ in detail and indicate how to prove the general case.

Proof. We assume that $Q$ is a $U(1) \times U(1)$ invariant distribution on $\mathbb{C} \times \mathbb{C} \times Y$. We also assume that $Q^{\tau}=-Q$. We will prove that $Q=0$.

Let $\mathbb{R}^{*}=\mathbb{R}-\{0\}, \mathbb{C}^{*}=\mathbb{C}-\{0\}$. We restrict $Q$ to $\mathbb{C}^{*} \times \mathbb{C}^{*} \times Y$ which is an open set. We define a map from $U(1) \times U(1) \times \mathbb{R}^{*} \times \mathbb{R}^{*} \times Y$ to $\mathbb{C}^{*} \times \mathbb{C}^{*} \times Y$ by

$$
\left(\lambda_{1}, \lambda_{2}, x_{1}, x_{2}, y\right) \mapsto\left(\lambda_{1} i x_{1}, \lambda_{2} i x_{2}, y\right), \quad \lambda_{1}, \lambda_{2} \in U(1), x_{1}, x_{2} \in \mathbb{R}^{*}, y \in Y .
$$

Here $i=\sqrt{-1}$. It is easy to check that this map is submersive onto $\mathbb{C}^{*} \times \mathbb{C}^{*} \times Y$. It induces a map from $C_{c}^{\infty}\left(U(1) \times U(1) \times \mathbb{R}^{*} \times \mathbb{R}^{*} \times Y\right)$ to $C_{c}^{\infty}\left(\mathbb{C}^{*} \times \mathbb{C}^{*} \times Y\right)$. Using the $U(1) \times U(1)$ invariance we can attach to $Q$ a distribution $\sigma_{Q}$ on $\mathbb{R}^{*} \times \mathbb{R}^{*} \times Y$. $\sigma_{Q}$ determines $Q$ and $\sigma_{Q}$ is skew invariant under the induced involution. It is easy to check that the induced involution is trivial on $\mathbb{R}^{*} \times \mathbb{R}^{*} \times Y$ hence $\sigma_{Q}=0$ and $Q=0$ on $\mathbb{C}^{*} \times \mathbb{C}^{*} \times Y$. (See the proof of (1], Lemma 4.2) for a more detailed explanation.)

We now restrict $Q$ to the open set $\mathbb{C} \times \mathbb{C}^{*} \times Y$. By our previous argument it follows that on this set $Q$ is supported on $0 \times \mathbb{C}^{*} \times Y$. Let $x_{1}+i y_{1}$ be coordinates on the first copy of $\mathbb{C}$. Then by a well known theorem of L. Schwartz, [14, there exist distributions $Q_{k, j}$ on $\mathbb{C}^{*} \times Y$ such that

$$
Q=\sum_{j, k \geq 0} \frac{\partial^{j}}{\partial x_{1}^{j}} \frac{\partial^{k}}{\partial y_{1}^{k}} Q_{j, k} .
$$


Here $Q_{j, k}=0$ for all but a finite number of indices $(j, k)$. Let $Z_{1}=\frac{\partial^{2}}{\partial x_{1}^{2}}+\frac{\partial^{2}}{\partial y_{1}^{2}}$. Since $Q$ is invariant under the action by $U(1)$ in the first component, it follows that there exist distributions $R_{j}$ on $\mathbb{C}^{*} \times Y$ and a positive integer $N$ such that

$$
Q=\sum_{j=0}^{N}\left(Z_{1}\right)^{j} R_{j} .
$$

Since the involution sends $\frac{\partial}{\partial x_{1}}$ to $-\frac{\partial}{\partial x_{1}}$ and $\frac{\partial}{\partial y_{1}}$ to $\frac{\partial}{\partial y}$, it follows that the involution fixes the differential operator $Z_{1}$. Hence the distributions $R_{j}$ are invariant under the action of $U(1)$, (the second $U(1)$ ) and skew invariant under the involution on $\mathbb{C} \times Y$. By the $n=1$ case it follows that $R_{j}=0$ for all $j$ hence $Q=0$ on $\mathbb{C} \times \mathbb{C}^{*} \times Y$. The same argument shows that $Q$ is zero on $\mathbb{C}^{*} \times \mathbb{C} \times Y$. It follows that $Q$ is supported on $0 \times 0 \times Y$.

We let $x_{2}+i y_{2}$ be coordinates on the second copy of $\mathbb{C}$ and $Z_{2}=\frac{\partial^{2}}{\partial x_{2}^{2}}+\frac{\partial^{2}}{\partial y_{2}^{2}}$. It follows that there exist distributions $R_{j, k}$ on $Y$ such that

$$
Q=\sum_{j, k \geq 0}\left(Z_{1}\right)^{j}\left(Z_{2}\right)^{k} R_{j, k}
$$

Since $\tau$ fixes $Z_{1}$ and $Z_{2}$, it follows that $Q^{\tau}=Q$. But we assumed that $Q^{\tau}=-Q$, hence $Q=0$.

The general case follows in the same way. The proof is by induction on $n$. We are given a distribution $Q$ on $\mathbb{C}^{n} \times Y$ which is $(U(1))^{n}$ invariant and satisfies $Q^{\tau}=-Q$. We restrict $Q$ to $\left(C^{*}\right)^{n} \times Y$ and show that it vanishes there. After that we perform $n$ steps. In the $k$ th step we restrict $Q$ to sets of the form $\mathbb{C}^{k} \times\left(\mathbb{C}^{*}\right)^{n-k} \times Y$ (after permutation) and use the induction assumption on $n-k$ to show that $Q$ vanishes on such sets.

5.1. Proof of Theorem [5.2. We shall prove Theorem 5.2 by induction on the semisimple rank of $H=H\left(n_{1}, \ldots, n_{k}\right)$. If the semisimple rank is zero, that is, $n_{i}=1, i=1, \ldots, k$, then we are in the situation of Theorem 5.3. So assume that the semisimple rank is positive. Let $Z(H)$ be the center of $H$. We will show that our distribution $Q$ on $H \times V \times Y$ is supported on $Z(H) \times V \times Y$. To do that we will need to show that $Q$ vanishes on every element $(h, v, y)$ such that $h \notin Z(H)$. Since every element in $H$ is conjugate to a diagonal element and since $Q$ is invariant under the action of $H$, it is enough to show that $Q$ vanishes on every element of the form $(s, v, y)$ where $s$ is diagonal and not in $Z(H)$. Let $s_{0}$ be such element and let $C$ be the centralizer of $s$. That is, $C=\left\{h \in H: h s_{0}=s_{0} h\right\}$. Then $C$ is block diagonal in $H$ and is isomorphic to $H\left(r_{1}, \ldots, r_{l}\right)$ for some positive integers $r_{1}, \ldots, r_{l}$. The semisimple rank of $C$ is smaller than the semisimple rank of $H$. Let $\mathfrak{c}$ be the Lie algebra of $C$ inside $\mathfrak{h}$, the Lie algebra of $H$. We can write $\mathfrak{h}=\mathfrak{c} \oplus B$ with $B$ an $A d(C)$ invariant subspace of $\mathfrak{h}$. (It is easy to describe $B$ in matrix form: $\mathfrak{c}$ is given by diagonal blocks in $\mathfrak{h}$ and $B$ is given by the off diagonal blocks that complement these blocks.) Set $C^{\prime \prime}=\left\{c \in C: \operatorname{det}\left((A d(c)-I)_{B}\right) \neq 0\right\}$. Set $\psi(h, c, v, y)=h(c, v, y)$ for $h \in H, c \in C^{\prime \prime}, v \in V, y \in Y$. Then $\psi$ is a submersion of $H \times C^{\prime \prime} \times V \times Y$ onto an open subset $U$ of $H \times V \times Y$. It is easy to see that $U$ is invariant under the action of $H$ and under the involution $\tau$. Since $s_{0}$ is in $C^{\prime \prime}$, it follows that the set $s_{0} \times V \times Y$ is in $U$. By Harish-Chandra's submersion principle ([16, 8.A.2.6) there is a one to one linear mapping between $H$ invariant distributions $Q$ on $U$ and $C$ invariant distributions $\tilde{Q}$ on $C^{\prime \prime} \times V \times Y\left(\tilde{Q}\right.$ is denoted by $\psi^{0}(Q)$ in [16], 8.A.3.2 (2)). 
Moreover, it is easy to check that a distribution $Q$ which is skew invariant under $\tau$ is mapped to a distribution $\tilde{Q}$ which is skew invariant under the restriction of $\tau$ to $C^{\prime \prime} \times V \times Y$. We would like to use the induction assumption to argue that such distributions $\tilde{Q}$ are identically zero. To do that we need to move from $C$ invariant distributions on $C^{\prime \prime} \times V \times Y$ to $C$ invariant distributions on $C \times V \times Y$. Let $\tilde{Q}$ be a distribution on $C^{\prime \prime} \times V \times Y$. Let $\phi \in C_{c}^{\infty}\left(\mathbb{R}^{*}\right)$. For a function $f \in C_{c}^{\infty}(C \times V \times Y)$ we attach a function $f_{\phi} \in C_{c}^{\infty}\left(C^{\prime \prime} \times V \times Y\right)$ by

$$
f_{\phi}(c, v, y)=f(c, v, y) \phi\left(\operatorname{det}\left((\operatorname{Ad}(c)-I)_{B}\right), \quad c \in C^{\prime \prime}, v \in V, y \in Y .\right.
$$

We define a distribution $\tilde{Q}_{\phi}$ on $C \times V \times Y$ by $\tilde{Q}_{\phi}(f)=\tilde{Q}\left(f_{\phi}\right)$. (The distributions $\tilde{Q}_{\phi}$ are approximating $\tilde{Q}$.) It is easy to see that if $\tilde{Q}$ is $C$ invariant, then $\tilde{Q}_{\phi}$ is $C$ invariant. It is easy to check that $\operatorname{det}\left((\operatorname{Ad}(c)-I)_{B}\right)$ is invariant under $\tau(c)=c^{t}$ hence if $\tilde{Q}$ is skew invariant under $\tau$, then $\tilde{Q}_{\phi}$ is skew invariant under $\tau$. By the induction assumption $\tilde{Q}_{\phi}=0$ for every $\phi \in C_{c}^{\infty}\left(\mathbb{R}^{*}\right)$. It follows that $\tilde{Q}=0$ and that $Q=0$. We have just proved that our original $Q$ vanishes on the open subset $U$ defined above hence on the set of elements $s_{0} \times V \times Y$.

Let $\mathfrak{s u}(n)=\left\{A \in M_{n}(\mathbb{C}): A^{t}=-A, \operatorname{tr}(A)=0\right\}$. Let $\mathfrak{s}=\mathfrak{s u}\left(n_{1}\right) \oplus \ldots \oplus \mathfrak{s u}\left(n_{k}\right)$ which we view as a Lie subalgebra of $\mathfrak{h}$. We let $\mathfrak{z}$ be the Lie algebra of $Z(H)$. Then $\mathfrak{h}=\mathfrak{z} \oplus \mathfrak{s}$. Let $U(\mathfrak{s})$ be the universal enveloping algebra of $\mathfrak{s}$ and $\zeta(\mathfrak{s})$ be the center of $U(\mathfrak{s})$. Let $s_{0} \in Z(H)$. Since $Q$ is supported on $Z(H) \times V \times Y$, it follows from the theory of distributions of L. Schwartz [14] (see Lemma 2.4 in [15] for the relevant formulation) that there exist an open set $U_{1}$ of $H$ around $s_{0}$ and differential operators $D_{1}, \ldots, D_{t} \in U(\mathfrak{s})$ such that

$$
Q=\sum_{j=1}^{t} L_{D_{j}} Q_{j}
$$

on $U_{1} \times V \times Y$. Here $Q_{j}$ are distributions on $\left(Z(H) \cap U_{1}\right) \times V \times Y$. Since both $\tau$ and $H$ fix $Z(H)$, we will move $Z(H) \cap U_{1}$ into $Y$ and view $Q_{j}$ as distributions on $V \times Y$. Moreover, if we write $D_{j}$ using a basis of $\mathfrak{s}$ as in [14] we get a unique expression for $Q$. Applying the action of $h \in H$ to $Q$ using the sum in (5.2) we get that

$$
Q=\sum_{j=1}^{t} L_{h\left(D_{j}\right)} h\left(Q_{j}\right)
$$

on the set $h\left(U_{1} \times V \times Y\right)=h U_{1} h^{-1} \times V \times Y$. Here $H$ acts via the Adjoint action on $\mathfrak{s}$ and $U(\mathfrak{s})$. $H$ acts on $V \times Y$ as above and consequently on distributions on $V \times Y$. Since $h s_{0} h^{-1}=s_{0}$ it follows that $h\left(U_{1} \times V \times Y\right) \cap\left(U_{1} \times V \times Y\right) \neq \emptyset$. Hence (5.2) is the same as (5.3) on the open set which is the intersection of these open sets. It follows from uniqueness that the action of $H$ fixes combinations of the differential operators appearing in (5.2). Hence there exist differential operators $E_{1}, \ldots, E_{l} \in \zeta(\mathfrak{s})$ so that

$$
Q=\sum_{j=1}^{l} L_{E_{j}} P_{j}
$$

on $U_{1} \times V \times Y$ where $P_{j}$ are distributions on $V \times Y$. The involution $\tau(h)=h^{t}$ induces an involution $\tau(A)=A^{t}$ on $\mathfrak{h}$ and on $\mathfrak{s}$. It is easy to see that $\tau$ stabilizes $\zeta(\mathfrak{s})$. We claim that $\tau$ fixes every element in $\zeta(\mathfrak{s})$. To see that, let $\mathfrak{c}$ be the diagonal Cartan 
subalgebra in $\mathfrak{s}$ and consider the Harish-Chandra isomorphism ([16, 3.2.3) from $\zeta(\mathfrak{s})$ to $U(\mathfrak{c})^{W}$. (Here $W$ is the Weyl group.) Then $\tau$ is moved by this isomorphism to an involution $\tilde{\tau}$ of $U(\mathfrak{c})^{W}$. By the explicit description of the Harish-Chandra isomorphism it follows that $\tilde{\tau}$ is obtained by restricting $\tau$ to $\mathfrak{c}$ and extending it to $U(\mathfrak{c})$. But $\tau$ fixes every element in $\mathfrak{c}$ hence in $U(\mathfrak{c})^{W}$.

We now apply $\tau$ to $Q$. By our assumption $Q^{\tau}=-Q$. On the other hand, applying $\tau$ to (5.4) we get that

$$
Q=\sum_{j=1}^{l} L_{\tau\left(E_{j}\right)} P_{j}^{\tau}=\sum_{j=1}^{l} L_{E_{j}} P_{j}^{\tau}
$$

on the set $\tau\left(U_{1} \times V \times Y\right)=\left(\tau\left(U_{1}\right) \times V \times Y\right)$. Here $P_{i}^{\tau}$ is a distribution on $V \times Y$ which is obtained by applying the involution $\tau(v, y)=(-\bar{v}, y)$ to $P_{i}$. Since $\tau\left(s_{0}\right)=s_{0}$, it follows that $\tau\left(U_{1} \times V \times Y\right) \cap\left(U_{1} \times V \times Y\right) \neq \emptyset$. Hence the expansions (5.4) and (5.5) are equal. By the uniqueness we get that $P_{j}^{\tau}=-P_{j}$. By the action of $H$ we get that $h P_{j}=P_{j}$ for every $h \in H$. Since $H \supset(U(1))^{m}$ where $m=n_{1}+\ldots+n_{k}$ we get that each $P_{j}$ satisfies the assumptions of Theorem 5.3. Hence $P_{j}=0, j=1, \ldots, l$ and $Q=0$ on $s_{0} \times V \times Y$. It follows that $Q=0$ and we are done.

\section{Distributions on the open Bruhat Cell}

Our strategy in the proof of Theorem 4.1 is to restrict our skew-invariant distribution $T$ to the open Bruhat cell and show that it vanishes there.

Let $X=P w P$ be the open Bruhat cell.

Proposition 6.1. Let $T$ be a distribution on $X$ and assume that $T$ satisfies (a) and (b) of Theorem 4.1 and that $T^{\tau}=-T$. Then $T=0$.

Proof. We define a map from $N \times P$ to $X=P w N$ by

$$
(n, p) \mapsto n p w n^{-1} \text {. }
$$

It is easy to check that this map is submersive hence by ([2], Lemma 2.3) it induces an onto mapping (which in this case is an isomorphism) from $C_{c}^{\infty}(N \times P)$ to $C_{c}^{\infty}(X)$. In particular, if $\alpha \in C_{c}^{\infty}(N)$ and $\beta \in C_{c}^{\infty}(P)$, then $\alpha \otimes \beta \in C_{c}^{\infty}(N \times P)$ is mapped to $f_{\alpha \otimes \beta} \in C_{c}^{\infty}(X)$ which is given by

$$
f_{\alpha \otimes \beta}(b w n)=\alpha(n) \beta(n b) .
$$

Since $T$ is invariant under conjugation by $N$, we get that there exist a distribution $\sigma_{T}$ on $P$ such that

$$
T\left(f_{\alpha \otimes \beta}\right)=\left(\int_{N} \alpha(n) d n\right) \sigma_{T}(\beta)
$$

for every $\alpha$ and $\beta$ as above. We will show that $\sigma_{T}=0$. Since $P$ is isomorphic to $N \times M$ via multiplication, it follows that we can identify $\sigma_{T}$ with a distribution which we again call $\sigma_{T}$ on $N \times M$. Since $T$ is invariant under conjugation by $S$ it follows that $\sigma_{T}$ is invariant the following action of $S$ on $N \times M$ :

$$
s(n, m)=\left(s n s^{-1}, s m s^{-1}\right), \quad s \in S, n \in N, m \in M .
$$

Since $T$ is skew invariant under $\tau$ it follows that $\sigma_{T}$ is skew invariant under $\tilde{\tau}$ where $\tilde{\tau}$ is given by

$$
\tilde{\tau}(n, m)=\left(\bar{n}^{-1}, \bar{m}^{-1}\right) .
$$


We identify $N$ with $\mathbb{C}^{n-1} \times \mathbb{R}$ in the following way: For $u \in \mathbb{C}^{n}$ and $x \in \mathbb{R}$ we let $n(u, x) \in N$ be defined by

$$
n(u, x)=\left(\begin{array}{ccc}
1 & u & -\bar{u}^{t} u / 2+x i \\
0 & I_{n-1} & -\bar{u} \\
0 & 0 & 1
\end{array}\right) .
$$

This mapping between $\mathbb{C}^{n-1} \times \mathbb{R}$ and $N$ is an isomorphism of manifolds. Thus we can identify $\sigma_{T}$ with a distribution $Q$ on $U(n-1) \times U(1) \times \mathbb{C}^{n-1} \times \mathbb{R}=U(n-1) \times$ $\mathbb{C}^{n-1} \times Y$ with $Y=U(1) \times \mathbb{R}$.

The invariance of $\sigma_{T}$ under $S$ implies the invariance of $Q$ under the action of $S$ on $U(n-1) \times \mathbb{C}^{n-1} \times Y$ given by

$$
d(1, X)(A, u, y)=\left(X A X^{-1}, X u, y\right), \quad X, A \in U(n-1), u \in \mathbb{C}^{n-1}, y \in Y .
$$

The skew invariance of $\sigma_{T}$ under $\tilde{\tau}$ implies that $Q$ is skew invariant under

$$
(A, u, y) \mapsto(-\bar{A},-\bar{u}, y) .
$$

Hence our proposition follows from Theorem [5.1.

\section{Distributions supported on the Closed Bruhat Cell}

Our strategy in the proof of Theorem 4.1 is to restrict the skew-invariant distribution $T$ to the open Bruhat cell and show that it vanishes there. After that we would like to show that invariant eigendistributions $T$ with support in the closed Bruhat cell vanish identically.

We shall need to define some elements in

$$
\mathfrak{g}=\mathfrak{u}(n, 1)=\left\{A \in M_{n+1}(\mathbb{C}): \bar{A}^{t} w+w A=0\right\} .
$$

Let $E_{j, k}$ be the $(n+1) \times(n+1)$ size matrix whose $(j, k)$ th entry is 1 and all other entries are 0 . We reserve the letter $i$ for $i=\sqrt{-1}$. Let $X_{j}=E_{1, j+1}-E_{j+1, n}$ and $Y_{j}=i\left(E_{1, j+1}+E_{j+1, n}\right), j=1, \ldots, n-1$. Let $Z=i E_{1, n+1}$. It is easy to check that all these elements are in $\mathfrak{g}$. Moreover, they form a basis for $\mathfrak{n}=\operatorname{Lie}(N)$. We let $\mathfrak{n}^{t}$ be the Lie subalgebra of $\mathfrak{g}$ obtained by taking transpose on all the elements of $\mathfrak{n}$. Then $X_{j}^{t}, Y_{j}^{t}, j=1, \ldots, n-1$ together with $Z^{t}$ form a basis for $\mathfrak{n}^{t}$. Let $\mathfrak{m}=\operatorname{Lie}(M)$ and let $U(\mathfrak{m})$ be the universal enveloping algebra of $\mathfrak{m}$. Let $\square$ be the Casimir element of $U(\mathfrak{g})$. Then there exist $D \in U(\mathfrak{m})$ so that

$$
\square=\sqrt{2} Z^{t} Z+\sum_{m=1}^{n-1} X_{m}^{t} X_{m}-\sum_{k=1}^{n-1} Y_{m}^{t} Y_{m}+D .
$$

Proposition 7.1. Let $T$ be a distribution on $G$ satisfying (a), (b), (c) in Theorem 2.4. Assume that $T$ is supported on $P$. Then $T=0$.

Proof. The crucial observations for this proof are the following. We first notice that by (b) of Theorem 2.4,

$$
L_{Z} T=c T
$$

for some nonzero $c \in \mathbf{C}$ depending on $\psi$. Also, using (a) of Theorem 2.4 we get that

$$
L_{X_{m}} T=R_{X_{m}} T, \quad L_{Y_{m}} T=R_{Y_{m}} T, \quad m=1, \ldots, n-1 .
$$

It will turn out to be essential to replace $L_{X_{m}}$ with $R_{X_{m}}$ and $L_{Y_{m}}$ with $R_{Y_{m}}$ as above. The reason is that $R_{X_{m}}$ commutes with all the differential operators $L_{A}$ for $A \in \mathfrak{g}$ while $L_{X_{m}}$ does not. 
We can now write the equation $L_{\square} T=\beta T$ in the form

$$
\sqrt{2} c Z^{t} T=\sum_{m=1}^{n-1}\left(L_{Y_{m}^{t}} R_{Y_{m}} T-L_{X_{m}^{t}} R_{X_{m}} T\right)+(\beta-D) T .
$$

Let $p \in P$. Since $T$ is supported on $P$ and since $\mathfrak{g}=\mathfrak{p} \oplus \mathfrak{n}^{t}$, it follows from the theory of distributions of L. Schwartz [14] that there exists an open set $U_{2}$ around $p$ such that

$$
T=\sum L_{Z^{t}}^{l} L_{Y_{1}^{t}}^{j_{1}} \ldots L_{Y_{n-1}^{t}}^{j_{n-1}} L_{X_{1}^{t}}^{k_{1}} \ldots L_{X_{n-1}^{t}}^{k_{n-1}} T_{l, J, K}
$$

on $U_{2}$. Here $J=\left\{j_{1}, \ldots, j_{n-1}\right\}, K=\left\{k_{1}, \ldots, k_{n-1}\right\}, T_{l, J, K}$ are distributions on $P$. Also, $T_{l, J, K}$ are determined uniquely and at most a finite number of them are nonzero. We shall think of the $T_{l, J, K}$ as the coefficients of the expression in (7.2) or the coefficients of $T$ at $p$. We notice that the distribution that appears in equation (7.1) is also supported on $P$ hence can be written around a neighborhood of $p$ as in (7.2) in a unique way. Our goal is to show that if $T$ is nonzero on $U_{2}$, then the left hand side and the right hand side of (7.1) yield different coefficients contrary to the uniqueness of (7.2). In particular, we will show that if $T \neq 0$ around $U_{2}$, then a certain coefficient of $Q=\sqrt{2} c Z^{t} T$ is nonzero on the left hand side of (7.1) while it is zero on the right hand side of (7.1). Write

$$
Q=\sum L_{Z^{t}}^{l} L_{Y_{1}^{t}}^{j_{1}} \ldots L_{Y_{n-1}^{t}}^{j_{n-1}} L_{X_{1}^{t}}^{k_{1}} \ldots L_{X_{n-1}^{t}}^{k_{n-1}} Q_{l, J, K}
$$

around $p$ as in (7.2). Then it is clear that

$$
Q_{l, J, K}=\sqrt{2} c T_{l-1, J, K}
$$

where we set $T_{l, J, K}=0$ if $l<0$. We now study the right hand side of (7.1). We first notice that if $A, B \in \mathfrak{g}$, then $L_{A}$ commutes with $R_{B}$. Hence we have

$$
\begin{gathered}
L_{X_{m_{0}}^{t}} R_{m_{j_{0}}} T=L_{X_{m_{0}}^{t}}\left(\sum L_{Z^{t}}^{l} L_{Y_{1}^{t}}^{j_{1}} \ldots L_{Y_{n-1}^{t}}^{j_{n-1}} L_{X_{1}^{t}}^{k_{1}} \ldots L_{X_{n-1}^{t}}^{k_{n-1}}\left(R_{X_{m_{0}}} T_{l, J, K}\right)\right), \\
L_{Y_{m_{0}}^{t}} R_{Y_{m_{0}}} T=L_{Y_{m_{0}}^{t}}\left(\sum L_{Z^{t}}^{l} L_{Y_{1}^{t}}^{j_{1}} \ldots L_{Y_{n-1}^{t}}^{j_{n-1}} L_{X_{1}^{t}}^{k_{1}} \ldots L_{X_{n-1}^{t}}^{k_{n-1}}\left(R_{Y_{m_{0}}} T_{l, J, K}\right)\right) .
\end{gathered}
$$

We notice that $R_{X_{m_{0}}} T_{l, J, K}$ and $R_{Y_{m_{0}}} T_{l, J, K}$ are some new distributions on $P$.

Let $\|J\|=j_{1}+\ldots+j_{n-1}$ and $\|K\|=k_{1}+\ldots+k_{n-1}$. We call $I(l, J, K)=$ $l+\|J\|+\|K\|$ the index of the coefficient $T_{l, J, K}$.

We now compare coefficients on both sides of (7.1). If $T \neq 0$ around $U_{2}$, then some coefficients $T_{l, J, K}$ are nonzero. We consider the nonzero coefficients for which their index is maximal. We will call them "maximal" coefficients. Among these "maximal" coefficients we pick one $\left(l_{0}, J_{0}, K_{0}\right)$ for which $l_{0}$ is maximal. It follows from (7.3) that $Q_{l_{0}+1, J_{0}, K_{0}} \neq 0$. However, we claim that on the right side of (7.1), $Q_{l_{0}+1, J_{0}, K_{0}}$ is zero which is a contradiction.

To show that, we claim that on the right side of (7.1), each nonzero coefficient $Q_{l, J, K}$ satisfies either $l<l_{0}+1$ or $l+\|J\|+\|K\|<l_{0}+1+\left\|J_{0}\right\|+\left\|K_{0}\right\|$. To see this we must compute the contributions of each summand in (7.4) and (7.5) and the contributions of $(\lambda-D) T$.

First we notice that the distribution $(\lambda-D) T$ does not contribute nonzero coefficients $Q_{l, J, K}$ with $l+\|J\|+\|K\|>l_{0}+\left\|J_{0}\right\|+\left\|K_{0}\right\|$. This follows from the fact that the bracket of an element $A \in \mathfrak{m}$ and an element $E$ of $\mathfrak{n}^{t}$ is an element of $\mathfrak{n}^{t}$. Hence when we commute $A$ across an element of the form $L_{Z^{t}}^{l} L_{Y_{1}^{t}}^{j_{1}} \ldots L_{Y_{n-1}^{t}}^{j_{n-1}} L_{X_{1}^{t}}^{k_{1}} \ldots L_{X_{n-1}^{t}}^{k_{n-1}}$, we never increase the size of $l+\|J\|+\|K\|$. 
We now compute the contributions of (7.4) (similarly with (7.5)). To do that we need to commute $L_{X_{m_{0}}^{t}}$ with the differential operators appearing before $L_{X_{m_{0}}^{t}}^{k_{m_{0}}}$ in each summand in order to get the unique expansion. However, $X_{m_{0}}^{t}$ commutes with the elements $Z^{t}, X_{j}^{t}, Y_{j}^{t}$ except for $Y_{m_{0}}^{t}$ for which we have $\left[X_{m_{0}}^{t}, Y_{m_{0}}^{t}\right]=-2 Z^{t}$. Hence $L_{X_{m_{0}}^{t}} L_{Y_{m_{0}}^{t}}=L_{Y_{m_{0}}^{t}} L_{X_{m_{0}}^{t}}-2 L_{Z^{t}}$. Using that it is possible to write explicitly the unique expression for

$$
L_{X_{m_{0}}^{t}}\left(L_{Z^{t}}^{l} L_{Y_{1}^{t}}^{j_{1}} \ldots L_{Y_{n-1}^{t}}^{j_{n-1}} L_{X_{1}^{t}}^{k_{1}} \ldots L_{X_{n-1}^{t}}^{k_{n-1}}\left(R_{Y_{m_{0}}} T_{l, J, K}\right)\right) .
$$

In each summand of the unique expression for (7.6) the index is less than or equal to $l_{0}+|| J_{0}||+|| K_{0} \|+1$. This is true because $l+|J|+|K| \leq l_{0}+|| J_{0}\left\|+|| K_{0}\right\|$ and applying $L_{X_{m_{0}}^{t}}$ can only increase the index by one. In order to get a nonzero coefficient of index $l_{0}+\left\|J_{0}\right\|+\left\|K_{0}\right\|+1$ we need to have $l+|J|+|K|=l_{0}+\left\|J_{0}\right\|+\left\|K_{0}\right\|$. In that case there will be exactly one coefficient with index $l_{0}+\left\|J_{0}\right\|+\left\|K_{0}\right\|+1$ which is

$$
L_{Z^{t}}^{l} L_{Y_{1}^{t}}^{j_{1}} \ldots L_{Y_{n-1}^{t}}^{j_{n-1}} L_{X_{1}^{t}}^{k_{1}} \ldots L_{X_{m_{0}}^{t}}^{k_{m_{0}}+1} \ldots L_{X_{n-1}^{t}}^{k_{n-1}}\left(R_{Y_{m_{0}}} T_{l, J, K}\right) .
$$

Since $l<l_{0}+1$, we get our conclusion.

Hence we get a contradiction and $T=0$ on $p$. Since $T$ is supported on $P$ we get that $T=0$.

7.1. Proof of Theorem 2.4. We are now ready to prove Theorem 2.4. Let $T$ be a distribution satisfying (a), (b), (c) of Theorem 2.4 and such that $T^{\tau}=-T$. We restrict $T$ to the open Bruhat cell $B w B$. By Proposition 6.1, $T=0$ on $B w B$. Hence $T$ is supported on $B$. By Proposition 7.1, $T=0$.

\section{REFERENCES}

1. Ehud Moshe Baruch, Ilya Piatetski-Shapiro, and Stephen Rallis, On the uniqueness of Fourier Jacobi models for representations of $\mathrm{U}(2,1)$, Lie groups and symmetric spaces, Amer. Math. Soc. Transl. Ser. 2, vol. 210, Amer. Math. Soc., Providence, RI, 2003, pp. 47-56. MR2018352 (2004k:22021)

2. Ehud Moshe Baruch and Steve Rallis, A uniqueness theorem of Fourier Jacobi models for representations of $\operatorname{sp}(4)$, J. London Math. Soc. (2) 62 (2000), no. 1, 183-197. MR.1772180 (2001j:22021)

3. S. Böcherer, J. H. Bruinier, and W. Kohnen, Non-vanishing of scalar products of FourierJacobi coefficients of Siegel cusp forms, Math. Ann. 313 (1999), no. 1, 1-13. MR1666805 (2000a:11066)

4. Stephen Gelbart, Examples of dual reductive pairs, Automorphic forms, representations and $L$-functions (Proc. Sympos. Pure Math., Oregon State Univ., Corvallis, Ore., 1977), Part 1, Proc. Sympos. Pure Math., XXXIII, Amer. Math. Soc., Providence, R.I., 1979, pp. 287-296. MR0546603 (81f:22035)

5. Stephen Gelbart and Ilya Piatetski-Shapiro, Automorphic forms and L-functions for the unitary group, Lie group representations, II (College Park, Md., 1982/1983), Springer, Berlin, 1984, pp. 141-184. MR0748507 (86f:11084)

6. Stephen S. Gelbart and Jonathan D. Rogawski, L-functions and Fourier-Jacobi coefficients for the unitary group U(3), Invent. Math. 105 (1991), no. 3, 445-472. MR.1117148|(93b:11059)

7. Miki Hirano, Fourier-Jacobi type spherical functions for $P_{J}$-principal series representations of $\operatorname{Sp}(2, \mathbf{R})$, J. London Math. Soc. (2) 65 (2002), no. 3, 524-546. MR1895731 (2003c:11046)

8. Yoshi-hiro Ishikawa, The generalized Whittaker functions for $\mathrm{SU}(2,1)$ and the Fourier expansion of automorphic forms, J. Math. Sci. Univ. Tokyo 6 (1999), no. 3, 477-526. MR1726680 (2001b:22011)

9. , The generalized Whittaker functions for the discrete series representations of SU(3,1), Sūrikaisekikenkyūsho Kōkyūroku (1999), no. 1094, 97-109, Automorphic forms on $\mathrm{Sp}(2 ; \mathbf{R})$ and $\mathrm{SU}(2,2)$, II (Kyoto, 1998). MR1751059 
10. Shin-ichi Kato, Atsushi Murase, and Takashi Sugano, Whittaker-Shintani functions for orthogonal groups, Tohoku Math. J. (2) 55 (2003), no. 1, 1-64. MR.1956080 (2003m:22020)

11. Jian-Shu Li, Minimal representations $\&$ reductive dual pairs, Representation theory of Lie groups (Park City, UT, 1998), IAS/Park City Math. Ser., vol. 8, Amer. Math. Soc., Providence, RI, 2000, pp. 293-340. MR 1737731 (2001a:22013)

12. Atsushi Murase and Takashi Sugano, Whittaker-Shintani functions on the symplectic group of Fourier-Jacobi type, Compositio Math. 79 (1991), no. 3, 321-349. MR1121142 (92k:11052)

13. _ Fourier-Jacobi expansion of Eisenstein series on unitary groups of degree three, J. Math. Sci. Univ. Tokyo 9 (2002), no. 2, 347-404. MR1904935 (2003f:11065)

14. L. Schwartz, Théorie des distributions. Tome I, Hermann \& Cie., Paris, 1950. MR0035918 $(12,31 \mathrm{~d})$

15. J. A. Shalika, The multiplicity one theorem for $\mathrm{GL}_{n}$, Ann. of Math. (2) 100 (1974), 171-193. MR0348047 (50:545)

16. Nolan R. Wallach, Real reductive groups. I, Academic Press Inc., Boston, MA, 1988. MR0929683 (89i:22029)

Department of Mathematics, Technion, Israel Institute of Technology, Haifa 32000 , ISRAEL

E-mail address: embaruch@math.technion.ac.il

Department of Mathematics, The Ohio State University, Columbus, Ohio 43210

E-mail address: haar@math.ohio-state.edu 